Accepted by The Astrophysical Journal

\title{
On the Near-Infrared Size of Vega
}

\author{
David R. Ciardi \\ 211 Space Sciences Building, Department of Astronomy, University of Florida, Gainesville, FL 32611 \\ ciardi@astro.ufl.edu \\ Gerard T. van Belle \\ Jet Propulsion Laboratory, California Institute of Technology, MS 171-113, 4800 Oak Grove, Pasadena, \\ CA 91109 \\ gerard@huey.jpl.nasa.gov \\ Rachel L. Akeson ${ }^{1}$ \\ Infrared Processing and Analysis Center, California Institute of Technology MS 100-22, 770 South Wilson \\ Avenue, Pasadena, CA, 91125 \\ rla@ipac.caltech.edu \\ Robert R. Thompson ${ }^{2}$ \\ Jet Propulsion Laboratory, California Institute of Technology, MS 171-113, 4800 Oak Grove, Pasadena, \\ CA 91109 \\ thompson@huey.jpl.nasa.gov \\ Elizabeth A. Lada \\ 211 Space Sciences Building, Department of Astronomy, University of Florida, Gainesville, FL 32611 \\ lada@astro.ufl.edu \\ and \\ Steve B. Howell \\ Astrophysics Group, Planetary Science Institute, Tucson, AZ 85705 \\ howell@psi.edu
}

\begin{abstract}
Near-infrared $(2.2 \mu \mathrm{m})$ long baseline interferometric observations of Vega are presented. The stellar disk of the star has been resolved, and the data have been fitted with a limb darkened stellar
\end{abstract}

\footnotetext{
${ }^{1}$ Jet Propulsion Laboratory, California Institute of Technology, MS 171-113, 4800 Oak Grove, Pasadena, CA 91109

${ }^{2}$ Department of Physics \& Astronomy, University of Wyoming, Laramie, WY 82071-3905
} 
disk of diameter $\Theta_{L D}=3.28 \pm 0.01$ mas. The derived effective temperature is $T_{\text {eff }}=9553 \pm 111$ $\mathrm{K}$. However, the residuals resulting from the stellar disk model appear to be significant and display organized structure. Instrumental artifacts, stellar surface structure, stellar atmosphere structure, and extended emission/scattering from the debris disk are discussed as possible sources of the residuals . While the current dataset cannot uniquely determine the origin of the residuals, the debris disk is found to be the most likely source. A simple debris disk model, with $3-6 \%$ of Vega's flux emanating from the disk at $r \lesssim 4 \mathrm{AU}$, can explain the residuals.

Subject headings: Circumstellar material - infrared: stars - stars: individual (Vega) - stars: fundamental parameters - techniques: interferometric

\section{Introduction}

Vega $(\alpha$ Lyrae $=$ HD $172167=$ HR $7001, \mathrm{~A} 0 \mathrm{~V}, \mathrm{~d}=7.76 \mathrm{pc})$ is arguably one of the most important stars in astrophysics. It has been used extensively as a spectrophotometric absolute flux standard in the optical, ultraviolet (Hayes 1985; Bohlin et al. 1990), and infrared (Cohen et al. 1992). Vega is one of the few main sequence stars for which an angular diameter measurement has been made $\left(\Theta_{L D}=3.24 \pm 0.07\right.$; Hanbury Brown, Davis, \& Allen (1974)), and Vega has been used as a template for our fundamental understanding and modeling of stellar atmospheres (e.g., Kurucz 1979; Dreiling \& Bell 1980; Castelli \& Kurucz 1994).

In addition to the importance of Vega for stellar astrophysics, Vega has been fundamental to our understanding of exo-zodiacal and extra-solar planetary systems. Aumann et al. (1984) reported that IRAS had detected an infrared excess above what was expected for the stellar photosphere at $\lambda \gtrsim 12 \mu \mathrm{m}$. The infrared excess has been attributed to a circumstellar disk believed to be a denser analog of our own zodiacal cloud.

Many questions regarding the debris disks around other stars remain unanswered. How much total mass is located within the disks? What is the morphology of the disks? What is the size distribution of the dust grains? What is the composition of the dust? What is the lifetime of the disks? Are the debris disks leftovers of active planet formation?

The intensity contrast between the stellar photosphere and the disk makes observing the disk at short wavelengths $(\lambda \lesssim 10 \mu \mathrm{m})$ difficult. As a result, most of the work on the debris disk properties of Vega (and other Vega-like sources) has been performed at far-infrared, submillimeter, and millimeter wavelengths. These observations have coarse spatial resolution, and the wavelengths are relatively insensitive to smaller, hotter grains located close to the star. Consequently, the data are biased to cooler and larger grains located in the outer portions of the debris disk $(r \gtrsim 50-100 \mathrm{AU})$, where the equilibrium temperature is $T \lesssim 100 \mathrm{~K}$ (Backman \& Paresce 1993), and comparatively little is known about the inner regions of debris disks $(r \lesssim 10$ $\mathrm{AU})$.

Dust grains located within a few AU of the star are expected to contribute most significantly at $\lambda \lesssim 10$ $\mu \mathrm{m}$, either through emission or scattering. Some Vega-like stars do indeed show near-infrared excesses with hot dust $(T \sim 500-1500 \mathrm{~K})$ located within a few AU of the star (e.g., Sylvester, Skinner, \& Barlow 1997). Vega-like sources with near-infrared excesses may be younger than sources that exhibit only longer wavelength $(\lambda \gtrsim 10 \mu \mathrm{m})$ excesses.

Previous work is inconclusive regarding the presence of a near-infrared excess associated with Vega. Stellar atmosphere models have consistently underestimated the measured infrared $(\lambda \gtrsim 2 \mu \mathrm{m})$ flux of Vega 
(Mountain et al. 1985; Leggett et al. 1986a). However, Leggett et al. (1986b) compared the near-infrared colors of Vega with $25 \mathrm{~B}$ and A stars and found the colors of Vega to be consistent with these stars within the uncertainties (a few percent). This led Leggett et al. (1986b) to suggest that perhaps stellar atmosphere models are incorrect and that the models underestimate the photospheric infrared flux of A-type stars. Bessell, Castelli, \& Plez (1998) comment it is difficult to understand where the current stellar atmosphere models for A-type stars fail, as the dominating $\mathrm{H}$ opacity in A stars is thought to be very well understood. Finally, Mégessier (1995) reviews the optical and near-infrared calibrations of Vega up through 1995 and critically examines the various calibration methods. Mégessier (1995) finds that Vega is apparently brighter in the near-infrared than a normal A0V star by $\sim 0.04 \mathrm{mag}(3.5 \%)$ at $\mathrm{K}$, with an increasing excess as the wavelength increases. Thus, as well studied as Vega is, it is unclear whether or not it has an excess at $\lambda \lesssim 10$ $\mu \mathrm{m}$.

If the disk is detectable, either as emission or scattering, at $\lambda \lesssim 10 \mu \mathrm{m}$, this would have important implications regarding the dust temperature, size distribution, composition, and overall morphology of the debris disk surrounding Vega, and Vega-like stars, in general. Unfortunately, the apparent near-infrared excess relative to current stellar atmosphere models is of the same order of magnitude as the flux calibration uncertainties (2-4\%; Leggett et al. (1986a)), and it is difficult to determine from photometry alone if Vega truly has a near-infrared excess.

In this paper, the possible near-infrared excess associated with Vega has been investigated via another method independent of photometry: infrared interferometry. Vega was observed over two seasons with the Palomar Testbed Interferometer (PTI, Colavita (1999)), in an attempt to ascertain if the debris disk around Vega is detectable by PTI at $2.2 \mu \mathrm{m}$. The interferometric experiment does do not rely upon precise infrared photometry, but rather on the capability of the interferometer to detect spatial structure (i.e., whether or not there is any extended emission beyond the stellar photosphere).

The details of the observations and data reduction are discussed in $\S 2$, and in $\S 3$, the results and analysis are discussed. Overall, the observed visibility curve is dominated by the resolved stellar disk (as expected). However, after modeling the data with a stellar photospheric disk, significant residuals, which display organized structure, remain. The possible sources of the residuals, including instrumental artifacts, stellar surface structure, stellar atmospheric structure, and extended emission/scattering from the debris disk, are explored.

\section{Observations and Data Reduction}

PTI is located at Palomar Observatory and is equipped with two $40 \mathrm{~cm}$ siderostats separated along a $110 \mathrm{~m} \mathrm{~N}-\mathrm{S}$ baseline. ${ }^{3}$ Observations of Vega were made in the $\mathrm{K}$ band $(2.2 \mu \mathrm{m})$ on 9 nights over two observing seasons: 1999 May 24, 25 \& November 4, and 2000 May 09 \& July 1, 4-6, 26. The K filter used is a good match to the CIT photometric system (Colavita 1999; Elias et al. 1982, 1983) and is sampled in five spectral channels $(R \sim 20)$ centered at $\lambda_{c}=2.009,2.106,2.203,2.299,2.396 \mu \mathrm{m}$. The spectral channel photons are sent through an optical fiber, which behaves like a spatial filter restricting the field of view of the fringe tracker to $1^{\prime \prime}$ (Gaussian FWHM). PTI is, therefore, effectively insensitive to emission on scales of several arcsec or larger. The fringe contrast or the squared visibility $\left(V^{2}\right)$ of the source brightness distribution projected on the sky is the resulting observable of the interferometer.

\footnotetext{
${ }^{3} \mathrm{PTI}$ can also be reconfigured for a $85 \mathrm{~m} \mathrm{~N}-\mathrm{W}$ baseline, not utilized in this experiment.
} 
Vega, along with calibration sources, was observed $2-5$ times during each night, and each observation was approximately 130 seconds long. The calibration of the Vega $V^{2}$ data, on a channel-to-channel basis, is performed by estimating the interferometer system visibility $\left(V_{s y s}^{2}\right)$ using calibration sources with model angular diameters and then normalizing the raw Vega visibility by $V_{s y s}^{2}$ to estimate the measured $V^{2}$ (Mozurkewich et al. 1991; Boden et al. 1998). Uncertainties in the system visibility and the calibrated target visibility are inferred from internal scatter among the data in a scan, the uncertainties associated with the predicted calibrator diameters, and standard error-propagation calculations.

The calibrators used are main sequence stars with predicted unresolved angular sizes of $<0.75$ mas: HD $166620(\mathrm{~K} 2 \mathrm{~V})$, HD $166014(\mathrm{~B} 9.5 \mathrm{~V})$, and HD $168914(\mathrm{~A} 7 \mathrm{~V})$. Calibrating the three calibration objects against each other produced no evidence of systematic errors, with all objects delivering reduced $V^{2}=1$. A summary of the calibrators is given in Table 1 .

The data collected on the various nights were sorted by projected baseline and wavelength and were averaged via a uncertainty weighted mean. The uncertainties of the means were estimated from the variance of the input data. The reduced dataset contains a set of four data points per spectral channel, and is displayed in Figure 1. The apparent discrete spatial frequency sampling is a direct result of the spectral sampling of the K-band filter. Vega transits nearly overhead at PTI; consequently, the projected baseline on the sky changes by only a few percent. Thus, the spatial frequency sampling $(B / \lambda)$ in Figure 1 is dominated by the spectral channels and not by changes in the projected baseline. Also shown in Figure 1 are separate uncertainty weighted means for the data collected in 1999 and the data collected in 2000 data.

In addition to the standard visibility calibrators, Altair was also observed on the same nights as Vega (only during the 2000 season). The Altair observations are described in detail by van Belle et al. (2001). In many ways, Altair is the near-perfect comparison star for Vega.

Altair is located in the same part of the sky as Vega, making it observable on the same nights. Altair is of similar spectral type (A7IV-V) to Vega which helps minimize comparison difficulties that may arise from differences in the spectral slopes across the broad $\mathrm{K}$ band filter. Altair is of similar brightness $(\mathrm{V}=0.77$, $\mathrm{K}=0.26 \mathrm{mag}$ ) and angular size (3.4 mas; van Belle et al. (2001)) to Vega. Because the signal-to-noise ratio of the observations is dependent upon both the brightness and the angular size of a source, the similar brightnesses and angular sizes yield similar data quality. Finally, Altair is not known to have an infrared excess (e.g., Dunkin, Barlow, \& Ryan 1997; Kuchner, Brown, \& Koresko 1998). Thus, the visibility curve for Vega can be compared to that of a resolved and uncontaminated stellar profile of similar spectral type, brightness, and angular size.

Altair is, however, rotating at a substantial fraction of its critical velocity and is viewed at a high inclination $\left(i \sim 40^{\circ}-60^{\circ}\right.$; Jordahl 1972; van Belle et al. 2001). As a result, Altair presents an (on-sky) elliptical stellar disk. In the analysis here, only the N-S baseline data for Altair has been utilized, which is relatively insensitive to the oblateness of the photosphere, and is well represented by a single angular diameter. A detailed summary of the interferometric data and analysis for Altair is presented by van Belle et al. (2001). van Belle et al. (2001) averaged the data over wavelength, but here, the wavelength information has been maintained to ensure a proper comparison to Vega.

The Altair data are shown in Figure 2. Because Altair is located at a declination which is $30^{\circ}$ lower than Vega, the magnitude of the projected baseline changes more significantly for Altair than for Vega. Thus, the changing projected baseline, coupled with the spectral sampling, results in a more uniformly distributed sampling of the spatial frequencies than what is associated with the Vega data. 


\section{Discussion}

\subsection{Apparent Stellar Disk}

The simplest interpretation of the data shown in Figure 1 is to assume that only the stellar photospheric disk contributes to the observed visibility function. A source which presents a uniform disk of angular size $\Theta_{U D}$ will yield a visibility curve of the form:

$$
V^{2}=\left[\frac{2 J_{1}\left(\pi(B / \lambda) \Theta_{U D}\right)}{\pi(B / \lambda) \Theta_{U D}}\right]^{2}
$$

where $J_{1}$ is the Bessel function of first order, $B$ is the magnitude of projected baseline vector, $\lambda$ is the wavelength of the observations, and $\Theta_{U D}$ is the apparent stellar uniform disk angular diameter. The visibilities were fitted under the assumption that the structure is independent of wavelength within the $\mathrm{K}$ filter.

The best fit angular diameter was found by evaluating equation (1) with a range of angular diameters $\left(\Theta_{U D}=0.1-10 \mathrm{mas}, \Delta \Theta_{U D}=0.001 \mathrm{mas}\right)$. The $\chi^{2}$ was calculated for each test value, and the best fit was determined by minimizing the $\chi^{2}$. The uncertainty was estimated via a Monte Carlo simulation where the data points were randomly adjusted by their individual uncertainties, and the data were re-fitted. The simulation was performed 5000 times, and the final uncertainty was estimated from the standard deviation of the best fit angular diameters. The best fit uniform disk diameter was found to be $\Theta_{U D}=3.24 \pm 0.01$ mas, with a reduced $\chi^{2} / \nu \sim 2.7$. Based upon the probability distribution of $\chi^{2}$, there is $<1 \%$ chance of exceeding such a large $\chi^{2} / \nu$.

While limb darkening in A stars at $2.2 \mu \mathrm{m}$ is expected to be relatively low (e.g. Claret 2000), assuming that the star is a simple uniform disk will cause an underestimation of the true, limb-darkened disk size of the star. Adapted from Hanbury Brown et al. (1974), the visibility function for a linear limb darkened stellar disk model can be parameterized as:

$$
V^{2}=\left[\frac{1-\mu_{\lambda}}{2}+\frac{\mu_{\lambda}}{2}\right]^{-2}\left[\frac{\left(1-\mu_{\lambda}\right) J_{1}\left[\pi(B / \lambda) \Theta_{L D}\right]}{\pi(B / \lambda) \Theta_{L D}}+\frac{\left(\mu_{\lambda}\right) j_{1}\left[\pi(B / \lambda) \Theta_{L D}\right]}{\pi(B / \lambda) \Theta_{L D}}\right]^{2}
$$

where $\mu_{\lambda}$ is the linear limb darkening coefficient $\left(\mu_{2.2 \mu \mathrm{m}} \approx 0.15\right.$ for Vega; Claret $\left.(2000)\right), j_{1}$ is the spherical Bessel function of first order, and $\Theta_{L D}$ is the apparent stellar limb darkened disk angular diameter. Hanbury Brown et al. (1974) estimate that a linear approximation to limb darkening has $<0.5 \%$ uncertainty in the determination of the stellar disk size, as long as the data sample the first lobe of the Bessel function (as our data do). A more complicated limb darkening model (e.g., a quadratic) could be applied; this would contribute additional Bessel function terms (both normal and spherical), but at ever decreasing contribution. The added terms most significantly alter the visibility function beyond the primary lobe (i.e., past our data sampling).

Evaluating $\Theta_{L D}$ over the range of $0.1-10$ mas $\left(\Delta \Theta_{L D}=0.001\right.$ mas $)$, equation (2) was sampled in a similar manner to equation (1). The best fit limb darkened stellar disk diameter was determined to be $\Theta_{L D}=3.28 \pm 0.01$ mas, with a reduced $\chi^{2} / \nu \sim 2.7$, no better than the uniform disk model.

The best uniform disk and limb darkened disk models are shown in Figure 1. On the scale of the plot, the two models are nearly indistinguishable. The observations sample the visibility curve along the first lobe of the Bessel functions; however, most of the power associated with limb darkening occurs near the first null and beyond (i.e., at higher spatial frequencies; Hanbury Brown et al. (1974)). Thus, our data are relatively insensitive to limb darkening, except as a matter of scaling (e.g., Hanbury Brown et al. 1974). The apparent 
angular diameter of the stellar disk for Vega is underestimated by a factor of $\sim 1.2 \%$, when a uniform disk model is assumed. The angular diameter measurement of Vega presented here represents the most precise size estimate of Vega to date. Previously, Hanbury Brown, Davis, \& Allen (1974) measured the $\lambda=0.44$ $\mu \mathrm{m}$ angular size of Vega and derived a limb darkened stellar diameter of $3.24 \pm 0.07$ mas, which agrees with our measurement within their uncertainties.

Coupled with knowledge of the bolometric flux of the star, the measured angular diameter yields the effective temperature:

$$
T_{\text {eff }}=2341\left[\frac{F_{b o l}}{\Theta_{R}^{2}}\right]^{1 / 4}
$$

where $F_{b o l}$ is the bolometric flux in units of $10^{8} \mathrm{erg} \mathrm{cm}^{-2} \mathrm{~s}^{-1}$, and $\Theta_{R}$ is the mean Rosseland (photospheric) angular diameter in mas. Estimating $\Theta_{R}$ with the derived $\Theta_{L D}$, the bolometric flux for Vega $\left(F_{b o l}=\right.$ $2983 \pm 120 \times 10^{8} \mathrm{erg} \mathrm{cm}^{-2} \mathrm{~s}^{-1}$; Alonso, Arribas, \& Martínez-Roger (1994)) yields an effective temperature of $T_{\text {eff }}=9553 \pm 111 \mathrm{~K} .{ }^{4}$ Recent atmosphere models by Castelli \& Kurucz (1994) indicate that Vega has a temperature in the range of $T_{\text {eff }}=9550-9650 \mathrm{~K}$.

While our results are in general agreement with previous work, the reduced $\chi^{2} / \nu \sim 2.7$ (for either of the stellar disk models) is not very good. The residuals (bottom panel of Figure 1) show that the uniform disk and limb darkened disk models overestimate the visibility at lower spatial frequencies and underestimate the visibility at higher spatial frequencies. If the residuals are a result of the underestimation of the limb darkening coefficient, increasing its value should decrease the residuals. In order to significantly improve the quality of the fit $\left(\chi^{2} / \nu \sim 1\right)$, the limb darkening coefficient would need to be an unrealistic $\mu_{\lambda} \gtrsim 1$. This value is 7 times larger the anticipated near-infrared limb darkening coefficient, and is twice as large as the optical coefficient. It is unlikely, therefore, that simply underestimating the limb darkening coefficient is the source of the residuals.

Over half of the data points are located $>1 \sigma$ away from the best fit models. But perhaps more suggestive is the fact that the residuals appear linear as a function of spatial frequency. A linear, uncertainty-weighted, least squares fit was performed to the residuals, which is shown in the bottom panel of Figure 1. The slope of the residuals is non-zero at the $6 \sigma$ level (slope $=0.0018 \pm 0.0003$ ), suggesting that the residuals shown in Figure 1 are significant. But from where do they originate?

\subsection{Instrumental Artifacts}

The first test was to determine if the residuals could be an artifact introduced into the data from either the hardware or the data reduction process. The 1999 data and the 2000 data were compared to search for possible systematic errors introduced by the hardware. In Figure 1, the 1999 data and the 2000 data, both averaged as a function of wavelength, are overplotted to determine if there was a systematic difference between the two observing seasons. The two data subsets agree extremely well with each other. In fact, their agreement is even more remarkable when it is noted that the optics for PTI were disassembled, re-coated, and re-assembled over the 1999-2000 winter. Given the agreement between the 1999 and 2000 data, and the fact that PTI is re-aligned nightly, it is difficult to understand how the residuals could be produced by the hardware in such a consistent manner.

\footnotetext{
${ }^{4}$ The uncertainty in the derived effective temperature is dominated by the uncertainty in the bolometric flux.
} 
A second possibility is that the residuals are introduced into the data during the reduction process. To test for this, the Altair data were reduced in a manner similar to how the Vega data were reduced. The Altair data are shown in Figure 2 with a best fit uniform disk of $\Theta_{U D}=3.42 \pm 0.02$ mas, and the residuals of the model fit are shown in the lower panel of Figure 2. The uniform disk fit for Altair is very good $\left(\chi^{2} / \nu \sim 0.8\right)$, and the model residuals do not appear to display the same organized structure as is seen in the Vega data. Thus, the same processing techniques did not appear to create the same type of residuals observed for Vega. Based upon the above analyses, it is difficult to understand the Vega model residuals in terms of instrumental effects.

\subsection{Stellar Surface Features}

While stellar spots are not expected on a hot star such as Vega, it is possible that surface features are present, and the stellar disk would no longer be illuminated uniformly. The observed visibility curve would then be altered, in comparison to a uniform disk or limb darkened disk model. It is conceivable, therefore, that the residuals are a result of star spots on the surface of Vega.

To test for this, the limb darkened disk plus star spot model developed by van Belle et al. (2001) was adapted and applied to the Vega data. The model is a limb darkened disk of diameter 3.28 mas with a randomly placed bright spot which covers $25 \%$ of the stellar surface. The resulting visibility curve is shown in Figure 3, and is compared to the data and the 3.28 mas LD curve from Figure 1.

The spots are on a smaller spatial scale than the stellar photospheric disk, thus contributing the majority of their power at spatial frequencies higher than where the data are located. That is, the spots affect mostly the location of the first null and the amplitude of the secondary lobes.

Near the location of the data, stellar surface features do affect the visibility curve on the scale of the residuals. However, as can be seen in the Figure 3, a single spot merely shifts the visibility curve without significantly changing the slope of the curve. Thus, a single spot can not reproduce the visibility curve observed for Vega. It is possible that a suite of spots in the correct combination could reproduce the residuals observed in the data.

The good agreement between the 1999 and 2000 data implies that the stellar surface features on Vega would have to persist over the period of 7 months or would have to reappear in such a way as to reproduce nearly the exact same visibility curve. So while, in principle, stellar surface features could produce the observed visibility curve residuals, this possibility is viewed as unlikely given the good agreement of the data separated by over 7 months.

\subsection{Stellar Atmosphere Structure}

As described in $\S 2$., the data spectrally sample the $\mathrm{K}$ band filter at 5 different wavelengths $(2.009,2.106$, $2.203,2.299, \& 2.396 \mu \mathrm{m}, \Delta \lambda=0.096 \mu \mathrm{m}$ ), but the $V^{2}$ data were fitted under the assumption that the stellar structure is independent of wavelength across the $\mathrm{K}$ filter. In principle, the data may be sampling different temperatures (layers) within the stellar atmosphere, and this might be the source of the residuals. To test for this, the limb darkened model in equation (2) was applied to the data as a function of wavelength.

For each of the five wavelengths within the data, it is assumed that the limb darkening coefficient is the 
same $\left(\mu_{\lambda}=0.15\right)$, and the apparent limb darkened stellar disks as a function of wavelength are derived. To ensure that the assumption of grey opacity across the $\mathrm{K}$ filter is an adequate approximation, an additional experiment was performed. As a function of wavelength, the limb darkening coefficient was adjusted until the derived stellar disk equaled (to within the uncertainties of the fitting) 3.28 mas, (the stellar diameter that best matched the dataset as a whole). The limb darkening fitting required a monotonically decreasing limb darkening coefficient with a full range of $\mu_{2.009 \mu \mathrm{m}}=0.20$ to $\mu_{2.396 \mu \mathrm{m}}=0.05$. However, for a 10,000 $\mathrm{K}$ star, a linear limb darkening coefficient of $\mu_{\lambda}=0.20$ corresponds to a wavelength between the J (1.25 $\mu \mathrm{m})$ and $\mathrm{H}(1.65 \mu \mathrm{m})$ photometric bands, and $\mu_{\lambda}=0.05$ corresponds to a wavelength $\lambda \gg 2.2 \mu \mathrm{m}$ (Claret, Díaz-Cordovés, \& Giménez 1995). Thus, the range of required limb darkening coefficients appears extreme, and the assumption of grey opacity across the $\mathrm{K}$ filter is deemed adequate for the test of whether atmospheric structure is being sampled by the interferometer.

The derived wavelength-dependent limb darkened stellar diameters are shown in Figure 4. At $2.009 \mu \mathrm{m}$, the apparent limb darkened disk diameter is $\Theta_{2.009 \mu \mathrm{m}}=3.24 \pm 0.03 \mathrm{mas}$, and at $2.396 \mu \mathrm{m}$, the apparent disk size is $\Theta_{2.396 \mu \mathrm{m}}=3.33 \pm 0.04$ mas - a size increase of $\sim 3 \%$ across the $\mathrm{K}$ band filter. The overall trend is represented by a linear fit of the form:

$$
\Theta_{L D}=(2.8 \pm 0.2 \mathrm{mas})+\left(0.21 \pm 0.01 \frac{\mathrm{mas}}{\mu \mathrm{m}}\right) \lambda .
$$

While the angular diameter trend across the $\mathrm{K}$ band filter is apparently linear, the parametric fit fails to predict reasonable wavelength-dependent limb darkened diameters in two ways. First, the linear relationship indicated by equation (4) predicts an optical $(\lambda=0.44 \mu \mathrm{m})$ limb darkened stellar diameter of 2.9 mas $5 \sigma$ smaller than the measured limb darkened diameter $\left(\lambda=0.44 \mu \mathrm{m}, \Theta_{L D}=3.24 \pm 0.07\right.$ mas; Hanbury Brown, Davis, \& Allen 1974). The optical size measurement of Vega should be sensitive to only the stellar photosphere. Dust emission at optical wavelengths is highly unlikely, and optical polarization studies have detected no polarization as would be expected for optical scattering (Mauron \& Dole 1998; Bhatt \& Manoj 2000). Second, the above linear relationship can not hold over all wavelengths as it predicts an infinite angular size at infinite wavelength. It is, of course, possible that because the data sample only a small range in wavelength, the relationship only appears to be linear, but it is difficult to reconcile such a linear progression across the $\mathrm{K}$ filter with the good agreement between the measured $0.44 \mu \mathrm{m}$ and $2.009 \mu \mathrm{m}$ angular diameters.

Is a $3 \%$ apparent size increase across the $\mathrm{K}$ filter even feasible for a $T_{\text {eff }} \approx 9600 \mathrm{~K}$ main sequence star? To answer this question, a NextGen stellar atmosphere model $\left(T_{\text {eff }}=9600 \mathrm{~K}, \log g=4.0,[\mathrm{M} / \mathrm{H}]=0.0\right)$ by Hauschildt, Allard, \& Baron (1999) was utilized. By assuming that at any given wavelength, the stellar atmosphere is probed to an optical depth of $\tau_{\lambda}=2 / 3$, the originating temperature layer within the star can be estimated by equating the observed flux $F_{\lambda}$ to $(2 / 3) B_{\lambda}(T)$. Convolving the spectral channels with the atmosphere model and solving for the temperature in an iterative fashion, the originating temperature is converted into a stellar radius via the temperature versus radius relationship of the atmosphere model. The flux across the $\mathrm{K}$ filter is found to arise from stellar radii located within $0.05 \%$ of each other - a value significantly smaller than the $3 \%$ implied by Figure 4 and equation (4).

The above analysis suggests that radial sampling of the stellar atmosphere across the K filter can not explain the observed visibility function. It is cautioned, though, that the models by Hauschildt, Allard, \& Baron (1999) are plane parallel atmosphere models, and Vega is suspected to be fast rotator $(v \sin i=245 \mathrm{~km}$ $\left.\mathrm{s}^{-1}\right)$, viewed nearly pole-on $\left(i \sim 5-6^{\circ}\right.$; Gulliver, Hill, \& Adelman 1994; Heinrichsen, Walker, \& Klass 1998), potentially complicating the above analysis. However, work by Jordahl (1972) indicates that a fast rotator 
viewed pole-on would appear merely as a heavily limb darkened stellar disk, and would not substantially affect the slope of the visibility curve at spatial frequencies located within the first null. An underestimated limb darkening coefficient as the source of the residuals was already explored in $\S 3.1$, and an unrealistic limb darkening coefficient $\left(\mu_{\lambda} \gtrsim 1\right)$ was required to reproduce the observed visibility function.

Finally, the Altair data do not appear to show the same residuals that are displayed in the visibility curve of Vega. As Altair is of slightly later spectral type (A7IV-V) than Vega (A0V), one might expect that atmospheric structure would be more evident in Altair than in Vega. This is apparently not the case indicating that either the residuals are not a result of atmospheric structure, or the nearly edge-on view of Altair $\left(i \sim 45^{\circ}-60^{\circ}\right.$; Jordahl (1972); van Belle et al. (2001)) has hidden the atmospheric structure.

Sampling of the radial stellar atmospheric structure across the K band filter appears unlikely as the source of the observed residuals, as this requires a $3 \%$ increase in the apparent stellar disk across the $\mathrm{K}$ filter alone. Further, the apparent stellar disk versus wavelength relationship predicts an optical size much smaller than what is observed. However, the stellar atmospheric structure, rotational velocity, and on-sky orientation of Vega are not fully understood (e.g., Castelli \& Kurucz 1994; Gulliver, Hill, \& Adelman 1994), and given the limited sample of the visibility function, atmospheric structure can not be fully excluded as the origin of the residuals.

\subsection{Debris Disk Contribution}

Current far-infrared and sub-millimeter observations indicate that the debris disk around Vega extends out to $20^{\prime \prime}-40^{\prime \prime}$, and is apparently viewed nearly face-on (Backman \& Paresce 1993; Heinrichsen, Walker, \& Klass 1998; Mauron \& Dole 1998). The disk contains a total mass of $M_{d} \sim 0.5 M_{M o o n}$, and has an infrared luminosity of $L_{I R} \sim f e w \times 10^{-3} L_{\odot}$ (Backman \& Paresce 1993). The disk has not previously been confirmed at wavelengths shorter $\lambda \lesssim 20 \mu \mathrm{m}$, but as discussed in $\S 1$, stellar atmosphere models have consistently underestimated the measured near-infrared $(\lambda \gtrsim 2-5 \mu \mathrm{m})$ flux of Vega. Altair does not have a detectable infrared excess at any wavelength (Cheng et al. 1992; Backman \& Paresce 1993; Kuchner, Brown, \& Koresko 1998), and the residuals in the Vega data are not matched in the Altair data. Can the debris disk be responsible for the residuals observed in the $2.2 \mu \mathrm{m}$ visibility function of Vega?

To test whether the debris disk can alter the stellar disk visibility function in such a way as to reproduce the observed curve, a simple model was created. The model consists of a central, limb darkened $\left(\mu_{\lambda}\right)$ star of angular diameter $\Theta_{L D}$, surrounded by a uniform intensity ring viewed face-on. The true disk is, of course, not likely to be uniformly illuminated, but adding a more elaborate intensity distribution increases the complexity of the model, which is already difficult to constrain with the current dataset. Additionally, more complex geometries introduce structure at higher spatial frequencies, and the data, located along the first lobe of the Bessel function, are largely insensitive to higher frequency complexities.

The model ring has an inner radius $r_{i}$ and an outer radius $r_{o}$, and it contributes a fraction $f$ to the total flux of the system. The uniform ring can be approximated by the subtraction of a smaller disk of angular diameter $\Theta_{i} \propto 2 r_{i}$ from a larger concentric disk of angular diameter $\Theta_{o} \propto 2 r_{o}$. The resulting visibility function is simply an addition of the components weighted by their relative flux contributions:

$$
V^{2}=\left[\frac{(1-f)}{\frac{1-\mu_{\lambda}}{2}+\frac{\mu_{\lambda}}{2}}\left(\frac{\left(1-\mu_{\lambda}\right) J_{1}\left[\pi(B / \lambda) \Theta_{L D}\right]}{\pi(B / \lambda) \Theta_{L D}}+\frac{\left(\mu_{\lambda}\right) j_{1}\left[\pi(B / \lambda) \Theta_{L D}\right]}{\pi(B / \lambda) \Theta_{L D}}\right)\right. \text { (Stellar Disk) }
$$




$$
\left.+2 f \frac{J_{1}\left(\pi(B / \lambda) \Theta_{o}\right)}{\pi(B / \lambda) \Theta_{o}}-2 f \frac{J_{1}\left(\pi(B / \lambda) \Theta_{i}\right)}{\pi(B / \lambda) \Theta_{i}}\right]^{2} \text { (Uniform Ring). }
$$

The outer radius $\left(r_{o}\right)$ of the model disk was set to 0.5 (4 AU), the HWHP radius of the field of view of the fringe tracker. The true disk extends well beyond this point, but PTI is insensitive to such extended emission. If the disk contributes to the visibility function, as measured by PTI, the disk must extend to within $\sim 4 \mathrm{AU}$ of the star. Modeling of the longer wavelength infrared excess $(\lambda \gtrsim 10 \mu \mathrm{m})$ does not require the presence of dust grains located so close to the star, but the observations also do not exclude such a possibility (Aumann et al. 1984; Backman \& Paresce 1993). The inner radius $\left(r_{i}\right)$ of the model ring was set by the radius at which the dust grains are expected to sublimate $\left(T_{d u s t} \sim 1500 \mathrm{~K}\right)$. Assuming pure blackbody grains, the sublimation radius of the grains can be estimated by

$$
r \approx L^{0.5}\left(\frac{278}{T_{d u s t}}\right)^{2} \mathrm{AU}
$$

where $r$ is in $\mathrm{AU}, T_{\text {dust }}$ is in $\mathrm{K}$, and $L$ is in $L_{\odot}$. For Vega, $L \approx 54 L_{\odot}$ which sets the inner radius of the disk to $r_{i} \sim 0.25 \mathrm{AU}\left(\Theta_{i} \sim 64\right.$ mas $)$.

The stellar disk $\left(\Theta_{L D}\right)$ was constrained by the temperature range of current atmosphere models for the stellar photosphere of Vega and equation (3). The highest estimate of the effective temperature for Vega is $T_{\text {eff }} \lesssim 9700 \mathrm{~K}$ (Dreiling \& Bell 1980), which corresponds to a stellar diameter limit of $\Theta_{L D} \gtrsim 3.19$ mas. Apart from this lower limit restriction on the stellar size, the stellar disk diameter $\left(\Theta_{L D}\right)$ and the fractional contribution $(f)$ of the disk (the only free parameters allowed) were adjusted $\left(\Delta \Theta_{L D}=0.01\right.$ mas, $\Delta f=0.5 \%)$ until the $\chi^{2}$ was minimized.

The best match to data was found with a stellar diameter of $\Theta_{L D}=3.20$ mas and a disk contribution of $f=5 \%$. The resulting visibility function is shown in Figure 5 where it is compared to the data and the $\Theta_{L D}=3.28$ mas limb darkened stellar disk. The resulting fit has a reduced $\chi^{2} / \nu \sim 1.4$ (LD disk model, $\left.\chi^{2} / \nu \sim 2.7\right)$. An estimate of the uncertainties associated with the model parameters was made by finding the models which yielded a $\chi^{2} / \nu \sim 1.7$. Based upon the probability distribution of $\chi^{2}$ and the number of degrees of freedom, there is approximately a $5 \%$ chance of exceeding such a $\chi^{2} / \nu$. The two "bounding" models have parameters of $\left[f=3 \%, \Theta_{L D}=3.22 \mathrm{mas}\right]$ and $\left[f=6 \%, \Theta_{L D}=3.2 \mathrm{mas}\right]$. These models are displayed in Figure 5.

While the star+ring model does not match perfectly to the data (and given the simplicity of the model, a perfect match was not anticipated), a small $5 \%$ flux contribution of the uniform ring has adjusted the slope of the visibility curve in precisely the manner required to reduce the residuals. In contrast to the limb darkened star model, the star+ring model does not overestimate and underestimate the visibility at lower and higher spatial frequencies.

With the disk parameters $\left(\Theta_{i} \& \Theta_{o}\right)$ fixed, the stellar diameter and the disk flux contribution are fairly well constrained. The disk radii were increased and decreased by a factor of 100 with no significant change in the results. Even if the inner radius of the disk is allowed to be at the stellar limb ( $~ 3.2$ mas $)$, a minimum disk contribution of 2-3\% is always needed to account for the observed residuals. If all of the parameters are adjusted simultaneously $\left(\Theta_{L D}, \Theta_{i}, \Theta_{o}, f\right)$, a "near perfect" solution can be found, but all of the parameters play against each other, and a single unique solution is not found. Interestingly, the star+ring model appears to be most sensitive to the flux contribution $f$ of the disk rather than the radii of the ring. This is likely an indication that the disk edges are "outside" the normal size sensitivity of PTI ( $\sim 4$ mas), but still detectable by the interferometer. 
Is this simplistic model realistic? The derived stellar diameter of 3.2 mas is in reasonable agree with the optical limb darkened diameter $\Theta_{L D}=3.24 \pm 0.07$ (Hanbury Brown, Davis, \& Allen 1974). As mentioned above, the optical size measurement of Vega should be sensitive to only the stellar photosphere. Using equation (3), the implied effective temperature for an angular diameter of 3.2 mas is $T_{\text {eff }}=9670 \mathrm{~K}$, within the range of currently accepted values for the effective temperature of Vega. Thus, the model yields a reasonable stellar diameter.

The $f=5 \%$ flux contribution of the debris disk is approximately a factor of two (2) larger than the excess predicted by atmosphere models (e.g., Mountain et al. 1985), and a factor of 1.5 larger than the excess deduced by Mégessier (1995). Thus, a $5 \%$ flux contribution, while not an outrageous requirement, is marginally larger than what is expected from current observations and models. However, the $f=3 \%$ lower limit for the infrared excess is certainly within the current photometric uncertainties of Vega. How much dust mass is required to produce a $3-6 \%$ infrared excess at $2.2 \mu \mathrm{m}$ ?

If it is assumed that 1) the grains emit as blackbodies, 2) the grains are at a single equilibrium temperature $\left.\left(T_{\text {dust }}\right), 3\right)$ the grains are spherical and the size distribution can be approximated with a single size $(a \sim 10-20 \mu \mathrm{m})$, and 4$)$ the average grain density is $\rho_{\text {dust }} \sim 3 \mathrm{~g} \mathrm{~cm}^{-3}$, the dust mass needed to produce a $3-6 \% 2.2 \mu \mathrm{m}$ excess can be estimated. A (upper limit) dust temperature of $T_{\text {dust }} \sim 1500 \mathrm{~K}$ implies a necessary dust mass of only $M_{\text {dust }} \sim 10^{-5} M_{\text {Moon }}$. If a lower temperature of $500-700 \mathrm{~K}$ is used (e.g., Sylvester, Skinner, \& Barlow 1997), a dust mass of $M_{\text {dust }} \sim 10^{-3} M_{\text {Moon }}$ is required. Aumann et al. (1984) suggested that as much as $10^{-3}$ of the emitting grain area (which is proportional to the grain mass for a single grain density and size) could be at $500 \mathrm{~K}$ and not violate the limits set by the IRAS observations. Thus, a rough estimate of the dust mass necessary to produce the inferred $2.2 \mu \mathrm{m}$ excess is within the limits of current models for the debris disk.

It is cautioned that the model of a stellar disk surrounded by a uniform ring is not intended to imply that the disk surrounding Vega is a uniform ring of material. The model was created to test whether extended structure around the central star could reasonably explain the observed residuals in the visibility function of Vega. More complete spatial frequency sampling is necessary to constrain a more complex (realistic) disk model. The presented results imply that the debris disk is a viable explanation.

\section{Summary}

Near-infrared $(2.2 \mu \mathrm{m})$ interferometric observations of Vega have been obtained. The resulting visibility data have been modeled with a $3.28 \pm 0.01$ mas diameter limb darkened stellar disk. After subtraction of the stellar disk model, residuals within the data appear organized and significant. Four possible sources of the residuals are discussed in detailed: instrumental artifacts, stellar surface features, stellar atmospheric structure, and the surrounding debris disk.

Emission/scattering from the debris disk is found to be the most likely explanation. A simple star + uniform ring model was developed to simulate the effect of the debris disk upon the visibility function. A $3-6 \%$ flux contribution from dust located within $r \lesssim 4 \mathrm{AU}$ can alter the stellar disk visibility function in precisely the manner that is observed. The interferometric observations appear to be mostly sensitive to the relative flux contribution of the disk and not to the geometry of the disk. If the source of the disk contribution is emission from warm dust, a (blackbody) dust mass of $\sim 10^{-3} M_{M o o n}$ at $T_{\text {dust }} \approx 500-700$ $\mathrm{K}$ could account for the apparent contribution. 
Our findings are very intriguing but inconclusive. Infrared polarization and/or infrared interferometric observations, which have better $u v$-plane coverage than those obtained with PTI, are required to verify and explain the results obtained here. The infrared capabilities of interferometers currently under construction (e.g., Keck Interferometer, CHARA, or the VLTI) would be invaluable to this project. If the debris disk has indeed been detected at $2.2 \mu \mathrm{m}$, this has important consequences towards our understanding of Vega, its debris disk, and debris disks in general.

DRC would like to thank Peter Hauschildt for very useful discussions regarding the structure of stellar atmospheres, and for kindly providing the NextGen stellar atmosphere models to the public. DRC was supported in part by NASA WIRE ADP NAG5-6751, and EAL acknowledges support from a Research Corporation Innovation Award and a Presidential Early Career Award for Scientists and Engineers to the University of Florida (NSF Grant \# 97-33367). Portions of this work were performed at the Jet Propulsion Laboratory, California Institute of Technology under contract with the National Aeronautics and Space Administration. The referee is thanked for adding to the clarity of this paper. A hearty thank you goes to the entire PTI collaboration for their efforts in maintaining and running a great interferometer (http://huey.jpl.nasa.gov/palomar/ptimembers.html). DRC and GvB would like to warn all PTI observers to be on the alert for giant inflatable bumblebees at 3 in the morning.

\section{REFERENCES}

Alonso, A., Arribas, S., \& Martínez-Roger, C. 1994, A\&A, 282, 684

Aumann, H. H. et al. 1984, ApJ, 278, L23

Backman, D. E. \& Paresce, F. 1993, in Protostars and Planets III, ed. E. H. Levy \& Lunine, J. I., University of Arizona Press, Tucson \& London, 1253

Bhatt, H. C., \& Manoj, P. 2000, A\&A, 362, 978

Bessell, M. S., Castelli, F., \& Plez, B. 1998, A\&A, 333, 231

Boden, A.F, et al., 1998, ApJ, 504, L39

Bohlin, R. C., Harris, A. W., Holm, A. V., \& Gry, C. 1990, ApJS, 73, 413

Castelli, F. \& Kurucz, R. L. 1994, A\&A, 281, 817

Cheng, K.-P., Bruhweiler, F. C., Kondo, Y., \& Grady, C. A. 1992, ApJ, 396, L83

Claret, A., Díaz-Cordovés, J., \& Giménez, A. 1995, A\&A, 114, 247

Claret, A. 2000, A\&A, 363, 1081

Colavita, M. M. et al. 1999, ApJ, 510, 505

Cohen, M., Walker, R. G., Barlow, M. J., \& Deacon, J. R. 1992, AJ, 104, 1650

Dreiling, L. A. \& Bell, R. A. 1980, ApJ, 241, 736

Dunkin, S. K., Barlow, M. J., \& Ryan, S. G. 1997, MNRAS, 286, 604 
Elias, J., Frogel, J., Matthews, K., Neugebauer, G., 1982, AJ, 87, 1029

Elias, J., Frogel, J., Hyland, A., Jones, T., 1983, AJ, 88, 1027

Gulliver, A. F., Hill, G., \& Adelman, S. J. 1994, ApJ, 429, L81

Hanbury Brown, R., Davis, J., \& Allen, L. R. 1974, MNRAS, 167, 121

Hanbury Brown, R., Davis, J., Lake, R. J. W., \& Thompson, R. J. 1974, MNRAS, 167, 475

Hauschildt, P. H., Allard, F., \& Baron, E. 1999, ApJ, 512, 377

Hayes, D. S. 1985, in Calibration of Fundamental Stellar Quantities, ed. D. S. Hayes, L. E. Passinetti, \& G. D. Phillip, Dordrecht, Reidel, 225

Heinrichsen, I., Walker, H. J., \& Klass, U. 1998, MNRAS, 293, L78

Jordahl, P. R. 1972, Ph.D. Thesis, University of Texas at Austin

Kuchner, M. J., Brown, M. E., \& Koresko, C. D. 1998, PASP, 110, 1336

Kurucz, R. L. 1979, ApJS, 40, 1

Leggett, S. K. et al. 1986a, A\&A, 159, 217

Leggett, S. K. et al. 1986b, MNRAS, 223, 443

Mauron, N. \& Dole, H. 1998, A\&A, 337, 808

Mégessier, C. 1995, A\&A, 296, 771

Mountain, C. M., Leggett, S. K., Selby, M. J., Blackwell, D. E., \& Petford, A. D. 1985, A\&A, 151, 399

Mozurkewich, D., et al., 1991, AJ, 101, 2207

Sylvester, R. J., Skinner, C. J., \& Barlow, M. J. 1997, MNRAS, 289, 831

van Belle, G. T., Ciardi, D. R., Thompson, R. R., Akeson, R. L. \& Lada, E. A. 2001, ApJ, in press 


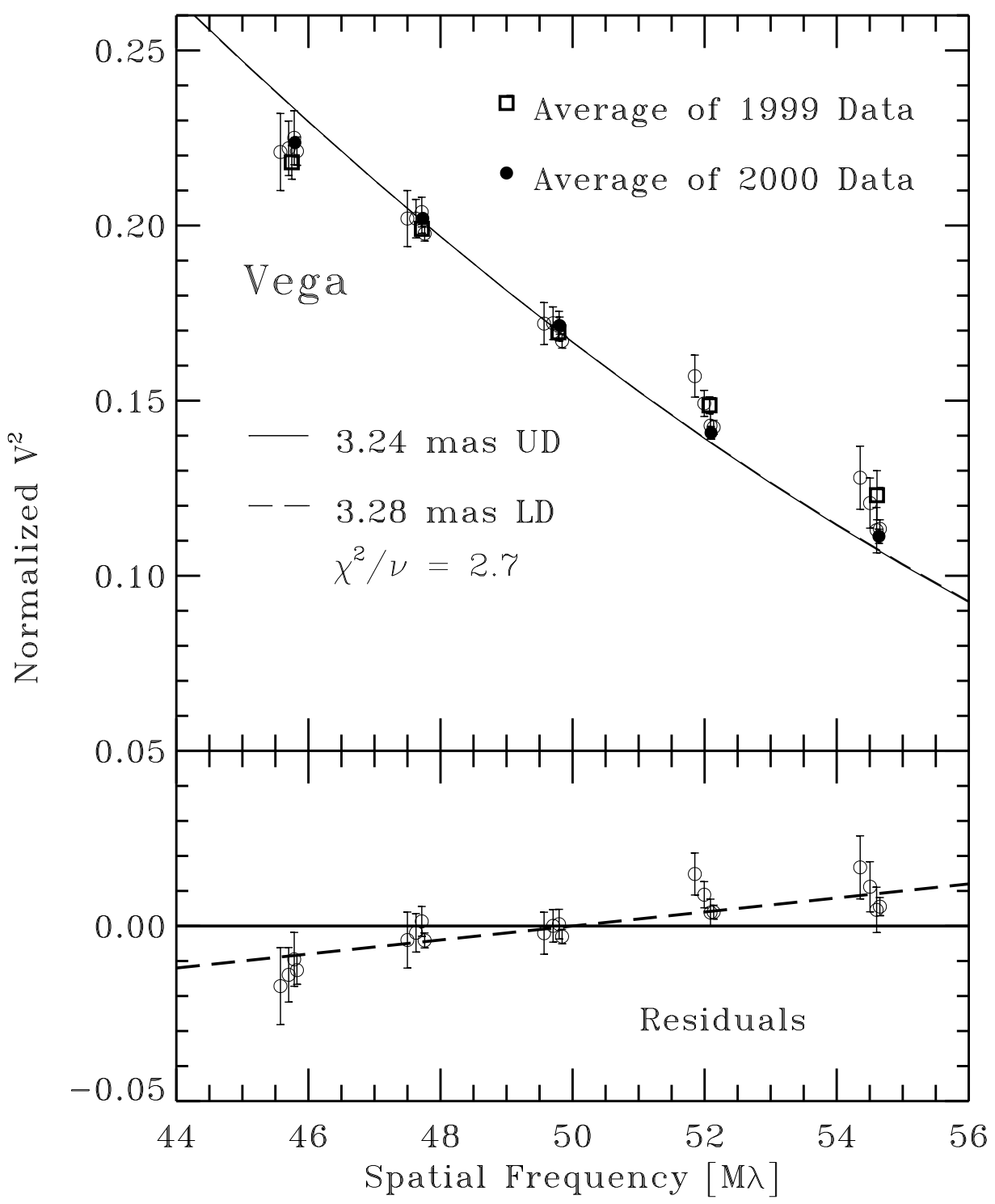

Fig. 1.- Top: Normalized visibility curve for Vega. The open circles represent data averaged as a function of baseline, regardless of year of acquisition. The 1999 data (open squares) and the 2000 data (filled circles) data, averaged by wavelength are shown. The $\Theta_{U D}=3.24$ mas uniform disk and the $\Theta_{L D}=3.28$ mas limb darkened disk models are overplotted and are nearly indistinguishable. Bottom: The residuals of the limb darkened disk model for the baseline averaged data are shown. The horizontal line marks the zero level, and the dashed line is a linear fit to the residuals. 


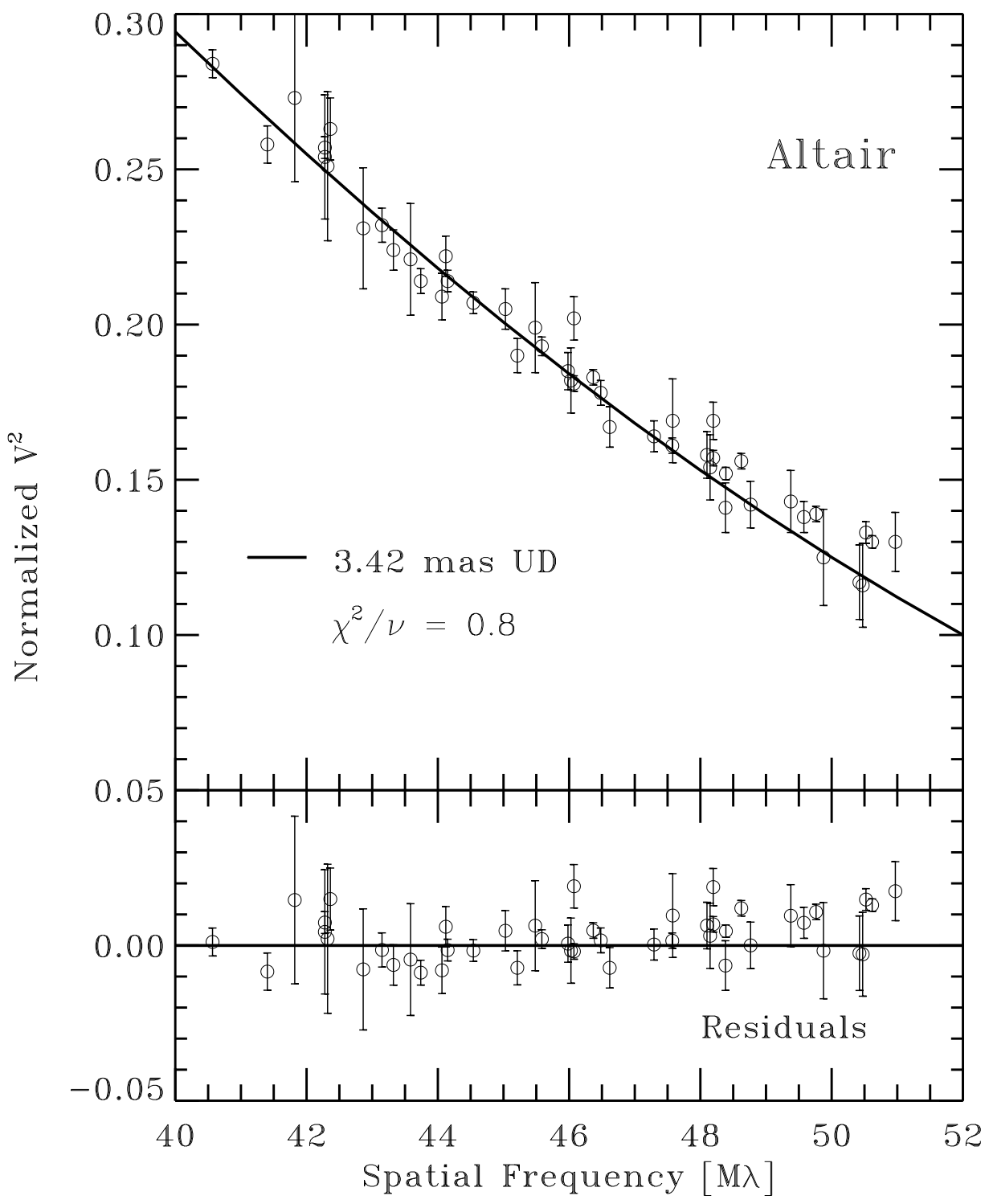

Fig. 2.- Top: Normalized visibility curve for Altair. The open circles represent data averaged as a function of baseline as was done for Vega (Figure 1). The $\Theta_{U D}=3.42$ mas uniform disk model is overplotted. Bottom: The residuals of the uniform disk model are shown. The horizontal line marks the zero level. 


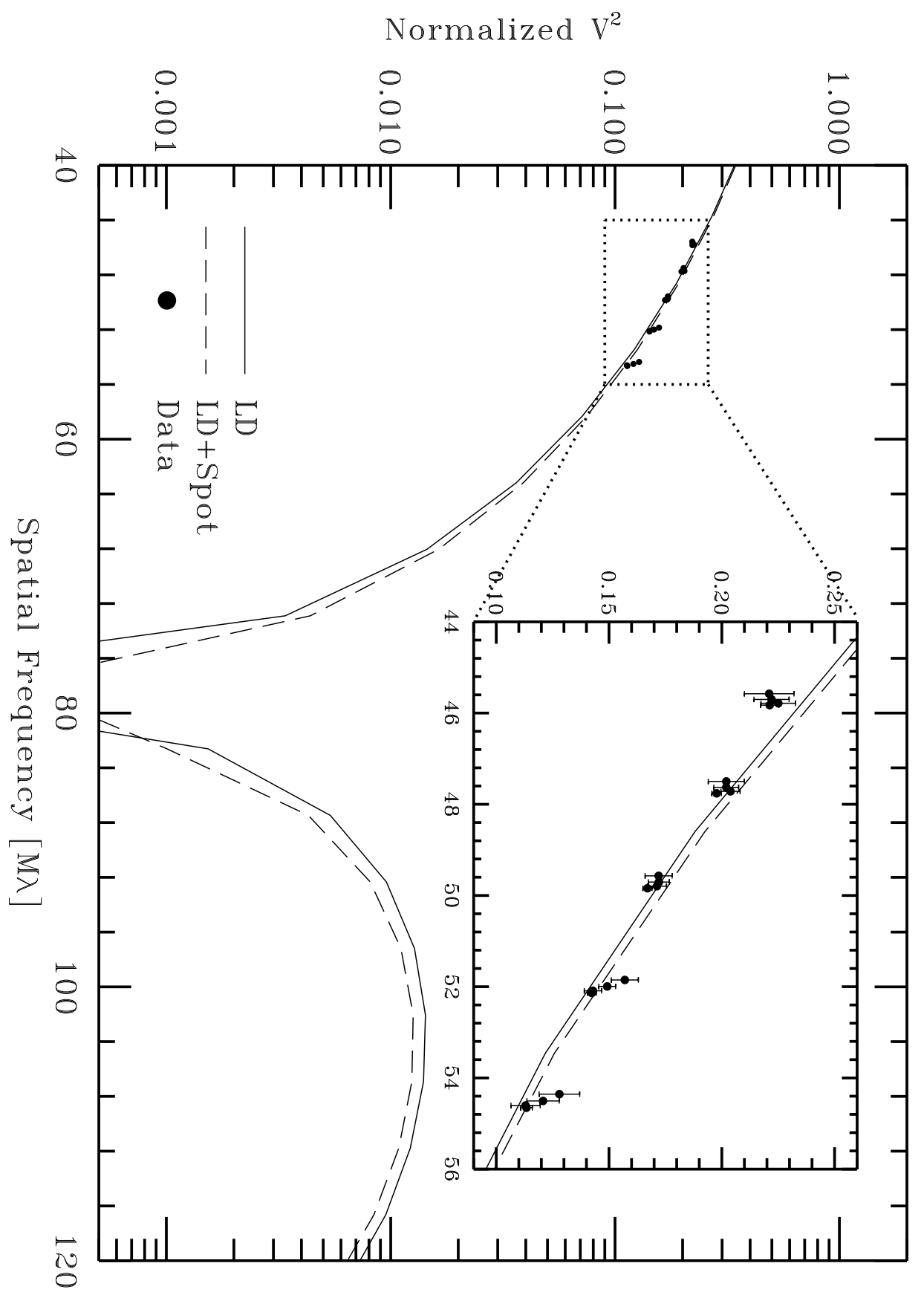

Fig. 3.- Normalized linear-log visibility curve for Vega. The solid curve represents the $\Theta_{L D}=3.28$ mas limb darkened model from Figure 1. The dashed curve represents the same limb darkened model, but with a surface spot covering $25 \%$ of the stellar disk. Inset: Detailed version of the plot with scaling set to that of the visibility curve shown in Figure 1. 


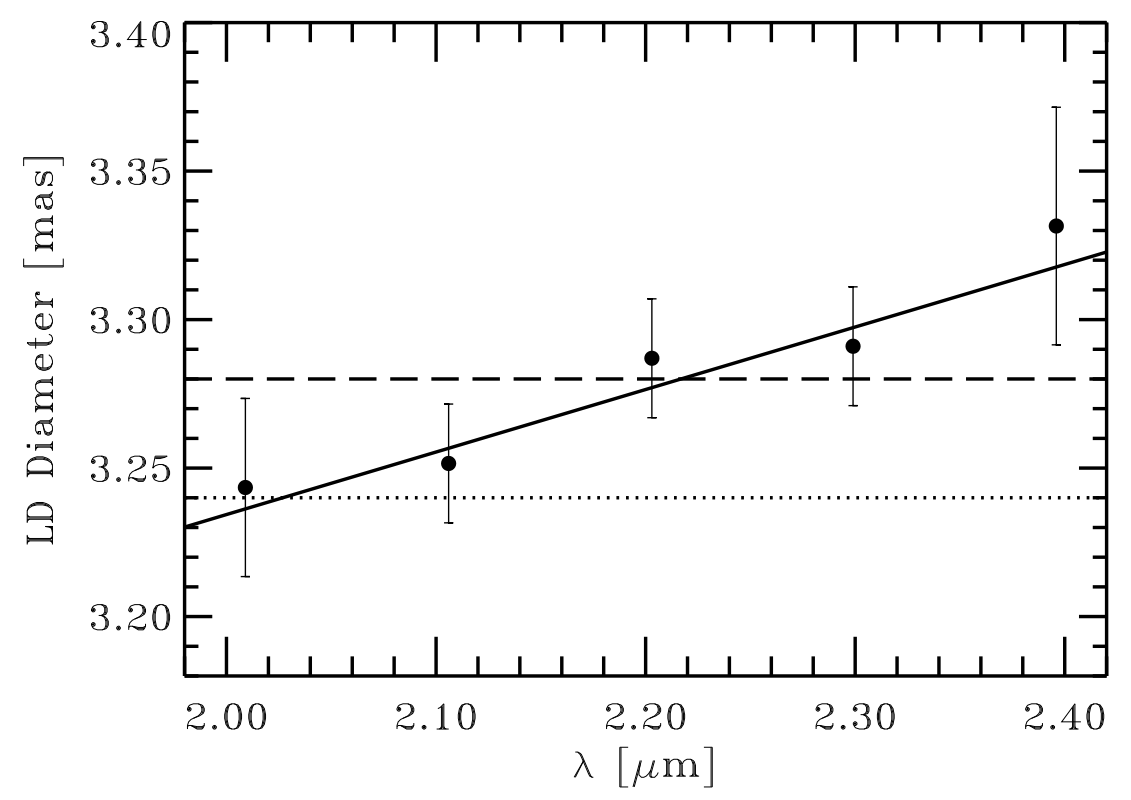

Fig. 4.- Apparent limb darkened disk sizes for Vega shown as a function of wavelength. The dashed line represents the angular diameter of the limb darkened model in Figure $1\left(\Theta_{L D}=3.28 \pm 0.01\right.$ mas), and the dotted line represents the limb darkened angular diameter obtained at $\lambda=0.44 \mu \mathrm{m}\left(\Theta_{L D}=3.24 \pm 0.07\right.$ mas; Hanbury Brown, Davis, \& Allen (1974)). 


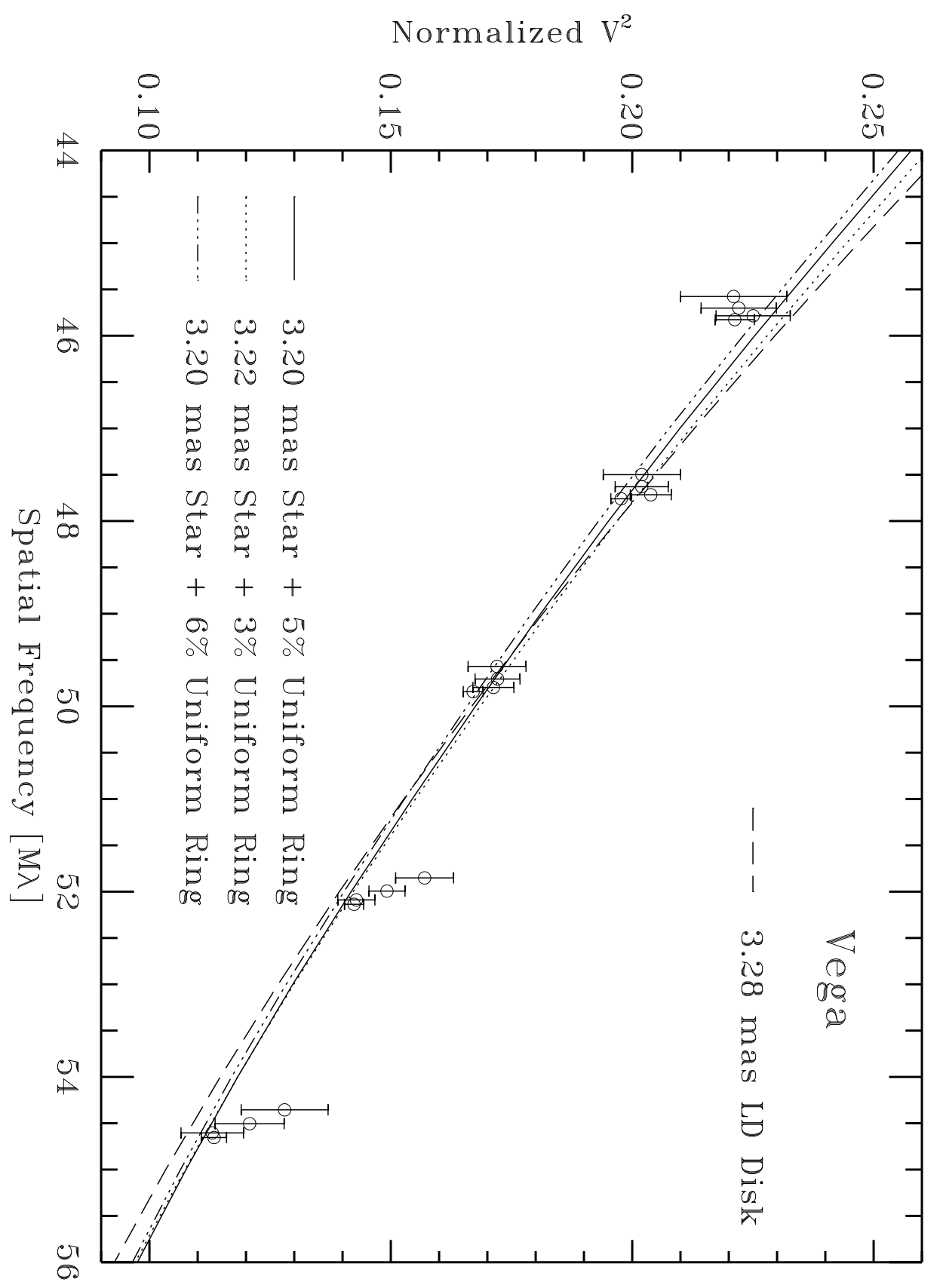

Fig. 5.- Normalized visibility curve for Vega. The dashed curve is the stellar limb darkened model from Figure 1. The solid curve represents the stellar disk $+5 \%$ uniform ring model $\left(\chi^{2} / \nu \sim 1.4\right)$. The dotted curve and the dash-dot curve represent estimated limits to the star + uniform ring models $\left(\chi^{2} / \nu \sim 1.7\right)$. 
Table 1. Calibration Sources.

\begin{tabular}{ccccc}
\hline \hline Source & $\begin{array}{c}\theta_{E S T^{\mathrm{a}}} \\
(\mathrm{mas})\end{array}$ & $\begin{array}{c}\text { Distance from } \\
\text { Vega }(\mathrm{deg})\end{array}$ & $\begin{array}{c}\text { Spectral } \\
\text { Type }\end{array}$ & Notes \\
\hline HD166620 & $0.74 \pm 0.08$ & 5.3 & K2V & Primary calibrator \\
HD166014 & $0.66 \pm 0.10$ & 11.7 & B9.5V & \\
HD168914 & $0.41 \pm 0.20$ & 10.4 & A7V & \\
\hline
\end{tabular}

${ }^{a}$ The estimated angular diameters come from photometric fits. 